\title{
Comparative study of monotonically convergent optimization algorithms for the control of molecular rotation
}

\author{
M. Ndong, M. Lapert, C. Koch ${ }^{\dagger}$ and D. Sugny ${ }^{\ddagger}$
}

February 28, 2022

\begin{abstract}
We apply two different monotonically convergent optimization algorithms to the control of molecular rotational dynamics by laser pulses. This example represents a quantum control problem where the interaction of the system with the external field is non-linear. We test the validity and accuracy of the two methods on the key control targets of producing molecular orientation and planar delocalization at zero temperature, and maximizing permanent alignment at non-zero temperature.
\end{abstract}

\section{Introduction}

Optimal control theory is nowadays a mature mathematical discipline with a wide range of applications in science and engineering [1. The technique has been used with success in quantum mechanics since the beginning of the 1990s [2, 3, 4, 5, to control spins, atoms and molecules by external electromagnetic fields. Control problems can be tackled by two different types of approaches, geometric [6, 7, 8] and numerical methods [9, 10, 11, 12, 13, 14, 15] for quantum systems of low and high dimension, respectively. It is this second aspect which is at the core of this article. Numerical optimal control algorithms can roughly be divided into Gradient ascent algorithms 12 and Krotov's method 10, 16, 20, 21, 17, 18, 19. The latter guarantees monotonic convergence independent of the specific choice of optimization functional, type of interaction between system and external control, and equations of motion. In the quantum control literature, Krotov's method was first established for dipole transitions, where the interaction of the system with the control field is linear [10, 21, 22, 23]. In recent years, several modifications to the known algorithms have been brought forward to account for the non-linear case, a problem which arises naturally in a variety of control problems in atomic and molecular physics. In particular it occurs when the intensity of the laser field is sufficiently large, so that the linear

\footnotetext{
*Institut für Quanteninformationsverarbeitung, Universität Ulm, D-89069 Ulm, GERMANY

${ }^{\dagger}$ Theoretische Physik, Universität Kassel, Heinrich-Plett-Str. 40, 34132 Kassel,Germany

¥Laboratoire Interdisciplinaire Carnot de Bourgogne (ICB), UMR 5209 CNRS-Université de Bourgogne, 9 Av. A. Savary, BP 47 870, F-21078 DIJON Cedex, FRANCE, dominique.sugny@u-bourgogne.fr
} 
model is no longer a good approximation of the dynamical system. While the generalization is straightforward for gradient algorithms, the extension of the monotonic approach is more involved [24, 25, 19]. Here, our goal is to explore the efficiency of two different schemes of monotonically convergent optimization algorithms for the control of a molecule interacting non-linearly with the control field.

The control of molecular rotation [26, 27, 28, 29, 30, 31, 32, 33, for which such non-linear models are well established [34, 35, is used as a testbed case to analyze the features of these algorithms. A first modification of a monotonically convergent algorithm to account for a non-linear interaction with the control assumes the cost to be quadratic in the field and decomposes the control into $n$ components for a nonlinearity of order $n$ 24. The decomposition leads to $2 n$ Schrödinger equations that need to be solved, $n$ for the wave function and $n$ for the adjoint state. This can be numerically costly. The approach was successfully applied to the control of molecular orientation and alignment [36]. At the same time, some of us proposed a new algorithm using only one component of the wave function 25. This comes at the price of changing the cost functional. Instead of penalizing the intensity of the field, i.e., the square of the control parameter, it penalizes a higher exponent, the value of which depends on the order of the nonlinearity. The algorithms of Refs. [24, 25] have recently been compared [37]. In the case of a two-color control strategy for molecular orientation, it was shown that the efficiency of the two optimized solutions designed by the two algorithms was similar. In parallel, it has been mentioned that the Krotov method allows for constructing a monotonically convergent algorithm with the standard cost functional penalizing the field intensity [19]. Here, we examine this claim and perform an extensive comparison with the Lapert algorithm [25]. We analyze the efficiency, numerical cost and structure of the optimized solutions obtained by the two approaches. The rotational dynamics of a diatomic molecule driven by an electromagnetic field will be used as an illustrative example.

The remainder of this paper is organized as follows. The molecular model is presented in Sec. 2. Section 3 is devoted to the application of the two algorithms to the control objectives of controlling molecular orientation and planar delocalization at zero temperature, and producing permanent alignment at non-zero temperature. We conclude in Sec.4 Appendix A summarizes briefly the two optimization algorithms.

\section{The model}

We consider the control of the rotational dynamics of the linear $\mathrm{CO}$ molecule described in a rigid rotor approximation and driven by the electric field $\vec{E}(t)$. The field is expressed as follows:

$$
\begin{aligned}
\vec{E}(t)= & \epsilon_{x}(t) \cos \left(\omega t+\Phi_{x}\right) \vec{e}_{x}+\epsilon_{z}(t) \cos \left(\omega t+\Phi_{y}\right) \vec{e}_{y}+ \\
& \epsilon_{z}(t) \cos \left(\omega t+\Phi_{z}\right) \vec{e}_{z}
\end{aligned}
$$

where $\epsilon_{\nu}(t), \vec{e}_{\nu}$, and $\Phi_{\nu}, \nu=x, y, z$ are the amplitude, the unit vector and the phase along the $\nu$-axis, respectively. The Hamiltonian of the system can be written as 34, 35]:

$$
H=B J^{2}+\vec{\mu} \cdot \vec{E}(t)+\alpha \cdot \vec{E}^{2}(t)+\beta \cdot \vec{E}^{3}(t),
$$




\begin{tabular}{|c|c|c|c|c|c|}
\hline$B\left(\mathrm{~cm}^{-1}\right)$ & $\mu_{0}$ (a.u.) & $\alpha_{\|}$(a.u.) & $\alpha_{\perp}$ (a.u.) & $\beta_{\|}$(a.u.) & $\beta_{\perp}$ (a.u.) \\
\hline 1.9312 & 0.112 & 15.65 & 11.73 & 28.35 & 6.64 \\
\hline
\end{tabular}

Table 1: Numerical values of the different molecular parameters.

where $B$ is the rotational constant. The first term of the right-hand side of Eq. (2) is the field-free rigid-rotor Hamiltonian. Its eigenstates are the spherical harmonics denoted by $|j, m\rangle$, with $j \geq 0$ and $|m| \leq j$. The operators $\vec{\mu}, \alpha$ and $\beta$ are associated, respectively, to the permanent dipole moment and the polarizability and hyperpolarizability tensors. The spatial position of the diatomic molecule is given in the laboratory frame by the spherical coordinates $(\theta, \phi)$.

We first study the interaction of the molecule with a non-resonant laser field, polarized linearly along the $z$-axis of the laboratory frame. In this case, the variable $\theta$ is the angle between the molecular axis and the polarization vector of the electric field. The Hamiltonian (2) then simplifies to

$$
\begin{aligned}
H= & B J^{2}-\mu_{0} \cos \theta E_{z}(t)-\frac{1}{2}\left[\Delta \alpha \cos ^{2} \theta+\alpha_{\perp} \mathbb{1}\right] E_{z}^{2}(t) \\
& -\frac{1}{6}\left[\left(\beta_{\|}-3 \beta_{\perp}\right) \cos ^{3} \theta+3 \beta_{\perp} \cos \theta\right] E_{z}^{3}(t),
\end{aligned}
$$

where $\Delta \alpha=\alpha_{\|}-\alpha_{\perp}$. The parameter $\mu_{0}$ is the permanent dipole moment and the coefficients $\alpha_{\|}, \alpha_{\perp}, \beta_{\|}$and $\beta_{\perp}$ denote, respectively, the polarizability and hyperpolarizability components of the molecule with the labels $\|$ and $\perp$ indicating the components parallel and perpendicular to the internuclear axis. The numerical values used in our simulations for the different molecular parameters are reported in Tab. 1. For details see Ref. 25. If we further assume that the frequency $\omega$ of the laser field is much higher than the rotational frequencies and non-resonant with respect to all rovibronic transitions, we can average over the fast oscillations of the electric field in Eq. (3) and obtain [35, 34]:

$$
\begin{aligned}
H(t)= & B J^{2}-\frac{1}{4}\left[\Delta \alpha \cos ^{2} \theta+\alpha_{\perp} \mathbb{1}\right] \epsilon_{z}^{2}(t) \\
& -\frac{1}{8}\left[\left(\beta_{\|}-3 \beta_{\perp}\right) \cos ^{3} \theta+3 \beta_{\perp} \cos \theta\right] \epsilon_{z}^{3}(t) .
\end{aligned}
$$

As a second example we consider the interaction of the $\mathrm{CO}$ molecule with a pulse that is elliptically polarized in the $(x, y)$-plane. We neglect here the hyperpolarizability term of the interaction since it does not play a quantitative role in this case. After optical-cycle averaging as above, the corresponding Hamiltonian is expressed as:

$$
\begin{aligned}
H= & B J^{2}-\frac{1}{4}\left[\left(\Delta \alpha \cos ^{2} \theta_{x}+\alpha_{\perp} \mathbb{1}\right) \epsilon_{x}^{2}(t)+\right. \\
& \left(\Delta \alpha \cos ^{2} \theta_{y}+\alpha_{\perp} \mathbb{1}\right) \epsilon_{y}^{2}(t)+ \\
& \left.2 \Delta \alpha \cos \left(\Phi_{x}-\Phi_{y}\right) \cos \theta_{x} \cos \theta_{y} \epsilon_{x}(t) \epsilon_{y}(t)\right],
\end{aligned}
$$

where $\cos \theta_{x}=\sin \theta \cos \varphi$ and $\cos \theta_{y}=\sin \theta \sin \varphi$.

In the case of zero rotational temperature $(T=0 \mathrm{~K})$, the time evolution of the system is described by the time-dependent Schrödinger equation,

$$
i \frac{\partial}{\partial t}|\psi(t)\rangle=H(t)|\psi(t)\rangle
$$


where $|\psi(t)\rangle$ is the wave function of the system at time $t$. The Liouville equation is used to describe the time evolution for $T \neq 0 \mathrm{~K}$ :

$$
i \frac{\partial}{\partial t} \rho(t)=[H(t), \rho(t)]
$$

where $\rho(t)$ represents the density matrix associated with the system at time $t$. Equations (6) and (7) are solved numerically with the split operator algorithm [38]. The Hamiltonian is represented in spherical harmonics $|j, m\rangle$ where all matrix elements are known analytically. We use atomic units unless otherwise specified.

\section{Numerical results}

We explore three different control targets presenting a comparative study of the Krotov and Lapert algorithms. The technical details of the algorithms are briefly reviewed in Appendix $\mathrm{A}$

\subsection{Orientation dynamics driven by a linearly polarized field}

We first investigate the control of molecular orientation by a field linearly polarized along the $z$-axis. In this case, the dynamics is described by Eq. (3). The control duration $t_{f}$ is chosen to be equal to one rotational period $T_{p e r}$ of the molecule, $T_{\mathrm{per}} \approx 8.6 \mathrm{ps}$. We consider a finite Hilbert space of size $j_{\max }=15$, which is sufficient for the intensity of the laser field used here. The expectation value $\langle\cos \theta\rangle$ is taken as a quantitative measure of the orientation. The molecule is oriented when $|\langle\cos \theta\rangle| \simeq 1$. Following Refs. [39, 40], we do not maximize this expectation value but a target state $\left|\psi_{f}\right\rangle$ maximizing $|\langle\cos \theta\rangle|$ in a sub-Hilbert space of finite dimension defined by $j \leq j_{f}$. The details of $\left|\psi_{f}\right\rangle$ can be found in Refs. 39, 40. Figure 1 shows the projection of the target state onto the eigenstates $|j, 0\rangle$ of the molecule, with $j_{f}=4$. A Gaussian pulse of 144 fs full width at half maximum (FWHM), centered at $t_{0}=T_{p e r} / 5$ is taken as guess field for all optimizations discussed in this section:

$$
E(t)=E_{0} e^{\frac{\left(t-t_{0}\right)^{2}}{2 \sigma^{2}}},
$$

where $\frac{1}{2} \epsilon_{0} c E_{0}^{2}=10^{12} \mathrm{~W} / \mathrm{cm}^{2}$ is the peak intensity of the laser field and the parameter $\sigma$ is defined such that FWHM $=2 \sqrt{2 \ln 2} \sigma$. This choice of guess field is standard in the control of molecular orientation [39. It provides an efficient initial solution with a population transfer from the state $|0,0\rangle$ to a superposition of $|j, 0\rangle$ states with $j=0,1, \cdots, j_{f}$. The cost functional is defined by:

$$
C=\left|\left\langle\psi_{f} \mid \psi\left(t_{f}\right)\right\rangle\right|^{2}-\lambda \int_{0}^{t_{f}}\left(E(t)-E_{r e f}(t)\right)^{n} / S(t) d t,
$$

where $n=2$ and $n=4$ for the Krotov and Lapert algorithms, respectively. The parameter $\lambda$ penalizes the pulse energy and $E_{r e f}(t)$ is a pulse reference. The function $S$, given by $S(t)=\sin ^{2}\left(\pi t / t_{f}\right)$, suppresses pulse amplitude at the beginning and end of the time window, ensuring a pulse that is smoothly switched on and off. We denote the final fidelity of the control by $C_{t_{f}}=\left|\left\langle\psi_{f} \mid \psi\left(t_{f}\right)\right\rangle\right|^{2}$. 


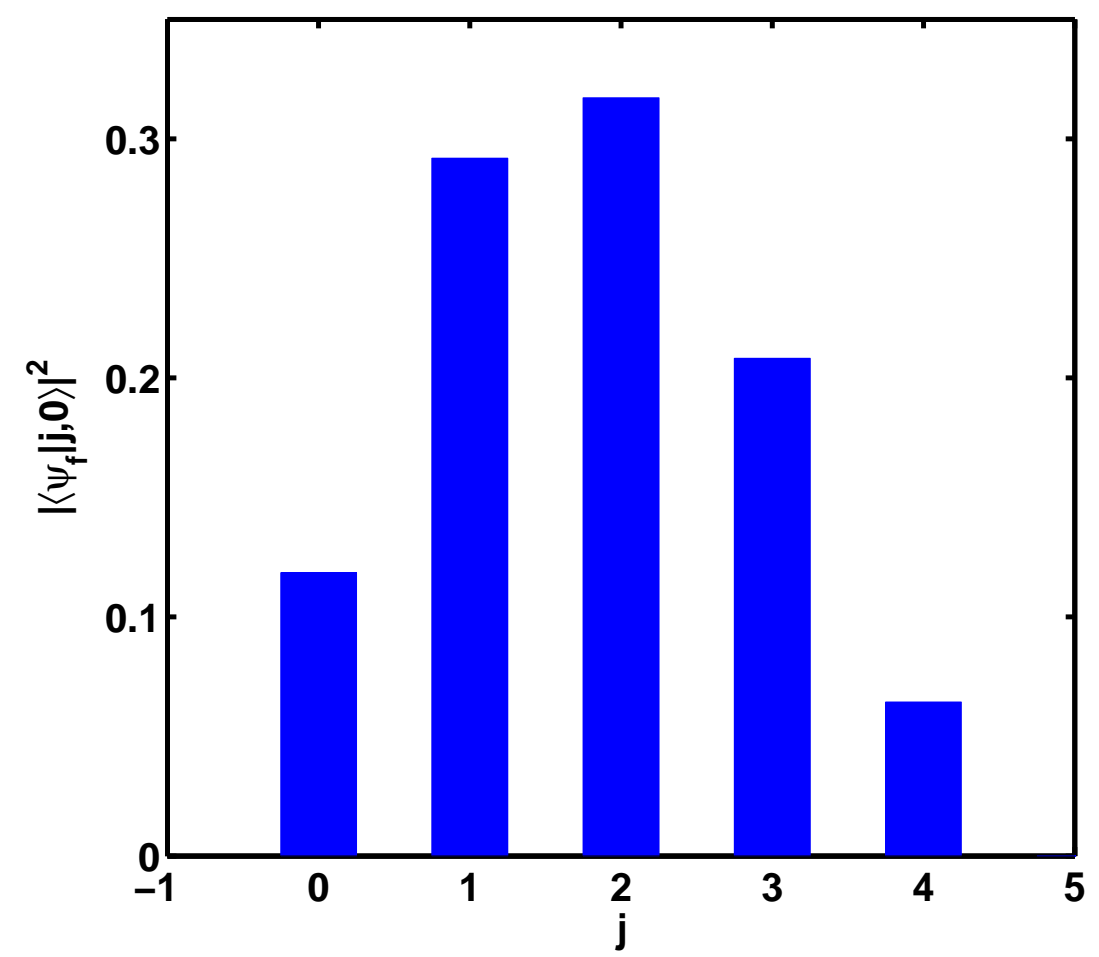

Figure 1: (color online) Population of the target state maximizing orientation of the $\mathrm{CO}$ molecule along the $z$-axis. The corresponding wave function is given by $\left|\psi_{f}\right\rangle \approx 0.34|0,0\rangle+0.54|1,0\rangle+0.56|2,0\rangle+0.46|3,0\rangle+0.25|4,0\rangle$

We first analyze the role of the parameter $\lambda$ in the two algorithms. The results reported in Tab. 2 show that the Krotov algorithm requires smaller $\lambda$ values to converge to a high fidelity $C_{t_{f}}$ than the Lapert method. In order to observe a convergence with realistic optimized pulses, the parameter $\lambda$ should be larger than $10^{2}$ for the Lapert method and lower than 0.1 for the Krotov one. This difference is easily understood from the fact that, in the Krotov formulation, the running cost is a quadratic function of the electric field, cf. Eq. (9), while in the algorithm by Lapert et al., this power is 4 . Since in atomic units, $\left|E(t)-E_{\text {ref }}\right|<1$, the same order of magnitude for the two running costs is obtained for $\lambda^{\mathrm{Lp}} \geq 10^{2} \lambda^{\mathrm{Kr}}$. Note that the parameter $\lambda^{\mathrm{Kr}}$ has to satisfy the relation (29) in order for the Krotov algorithm to be monotonic. The fact that there is no constraint in the choice of $\lambda^{\mathrm{Lp}}$ allows more flexibility in the use of the Lapert algorithm. However, one should keep in mind that large values of $\lambda^{\mathrm{Lp}}$ are required in order to avoid fast oscillations in the optimized solution. In this section, the two coefficients will be fixed at $\lambda^{\mathrm{Lp}}=5 \times 10^{6}$ and $\lambda^{\mathrm{Kr}}=5 \times 10^{-2}$. The evolution of the final fidelity $C_{t_{f}}$ as a function of the number of iterations and the CPU time is displayed in Fig. 2. While the Lapert formulation converges faster initially, the insets of Fig. 2 show that the Krotov algorithm becomes faster when the fidelity is close to 1 . 


\begin{tabular}{|c|c|c|c|c|}
\hline$\lambda$ & $C_{t_{f}}^{\mathrm{lp}}$ & $C_{t_{f}}^{\mathrm{kr}}$ & $E_{\max }^{\mathrm{lp}}$ & $E_{\max }^{\mathrm{kr}}$ \\
\hline $510^{6}$ & 0.9892 & 0.0205 & $5.510^{-3}$ & $5.510^{-3}$ \\
$510^{4}$ & 0.9993 & 0.0205 & $5.510^{-3}$ & $10^{-2}$ \\
$510^{2}$ & 0.9996 & 0.0205 & $5.510^{-3}$ & $2.810^{-2}$ \\
$510^{-1}$ & -- & 0.5789 & -- & $5.510^{-3}$ \\
$510^{-2}$ & -- & 0.9959 & -- & $5.510^{-3}$ \\
$510^{-3}$ & -- & 0.9944 & -- & $5.510^{-3}$ \\
\hline
\end{tabular}

Table 2: Analysis of the convergence of the two algorithms with respect to the parameter $\lambda . C_{t_{f}}^{\mathrm{lp}}$ and $C_{t_{f}}^{\mathrm{kr}}$ are the fidelities obtained from Krotov and Lapert algorithms, respectively. $E_{\max }^{\mathrm{lp}}$ and $E_{\max }^{\mathrm{kr}}$ correspond to the maximum amplitude in absolute value of the two optimized solutions. The number of iterations is set to 20 . In the Lapert algorithm, small values of $\lambda$ lead to very fast oscillations of the corresponding optimized field, which are not physically relevant. Therefore results are not indicated in columns 2 and 4 when $\lambda$ is smaller than $5 \times 10^{2}$.

The Lapert algorithm is however more costly in terms of computer time (CPU time). The faster convergence of Krotov's method in terms of CPU time is not surprising since in the Lapert formulation, at each iteration, the roots of a polynomial of power 3 need to be determined in order to compute the updated new pulse, see the appendix $\mathrm{A} .2$ for details. Figure 3 compares the optimized pulses obtained from the Lapert algorithm, Fig. 3h and from the Krotov one, Fig. 3k. Figure 3b displays the guess field considered for the two algorithms. Note that the structure of the Krotov solution is very simple, since the optimized field is mainly composed of the guess pulse plus additional small deformations. The solution designed by the Lapert algorithm is rather more complex, in the sense that fast oscillations appear between the middle and the end of the optimization time interval. As already pointed out in [25], this behavior seems to be quite general with a cost functional penalizing the power 4 of the field. Note that spectral filters can be added to avoid such oscillatory structures [41, 42].

The dynamics induced by the two optimized pulses is plotted in Fig. 4. More precisely, figure 4 displays the projection of the wave function onto the molecular eigenstates as a function of time. The two dynamics show similar features in the first fifth of the optimization time interval, $\left[0,0.2 \times t_{f}\right]$. Most of the population remains in the ground state, $|j=0, m=0\rangle$, since, in this time interval, both optimized pulses are almost zero. At time $t=0.2 \times t_{f}$, both optimized solutions contain a kick which leads to a superposition of states with $j=0, \ldots, 3$. For the dynamics induced by the Krotov optimized pulse, we observe that most of the population is concentrated in states $|j=3,0\rangle$ and $|j=4,0\rangle$ during the time interval $\left[0.4 \times t_{f}, 0.6 \times t_{f}\right]$, while these states are populated for $t \in\left[0.6 \times t_{f}, 0.8 \times t_{f}\right]$ in the Lapert case. In the time interval $\left[0.6 \times t_{f}, 0.8 \times t_{f}\right]$, the dynamics induced by the Krotov optimized field shows a superposition of states $|1,0\rangle,|2,0\rangle$ and $|3,0\rangle$, more or less similar to the target state. In the final step, i.e., in the time interval $\left[0.8 \times t_{f}, t_{f}\right]$, the small oscillations of the Lapert and Krotov fields are responsible for the complete transfer of the superpositions to the target state. 

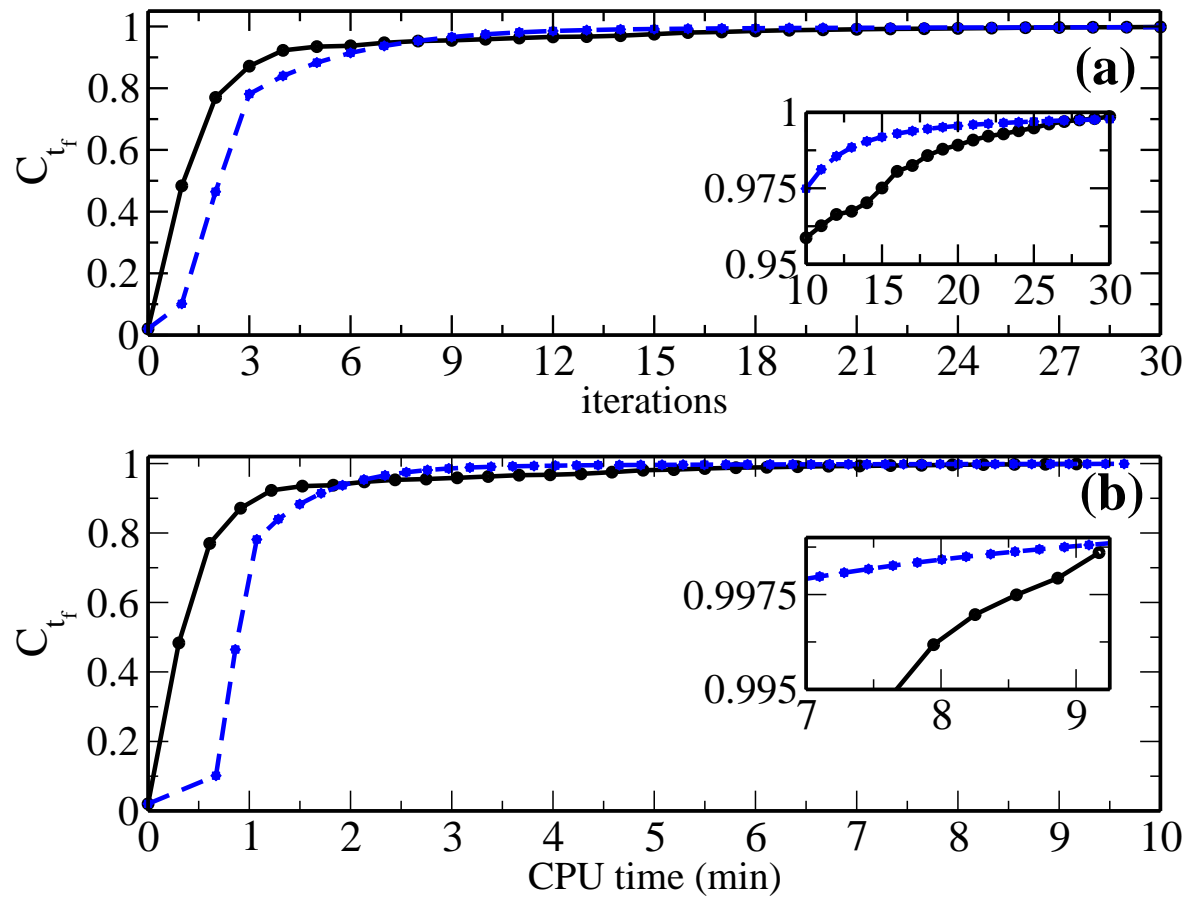

Figure 2: (color online) Convergence of the Lapert and Krotov algorithms measured by the final cost $C_{t_{f}}$ plotted as a function of the number of iterations (top panel) and $C_{t_{f}}$ plotted as a function of the CPU time (bottom panel). The results for the Lapert formulation are depicted in black solid line with circles, while a blue dashed line with stars represents the results with the Krotov algorithm.

\subsection{Delocalization in the $(x, y)$ - plane}

The second example is dedicated to controlling the orientation of the angular momentum of the $\mathrm{CO}$ molecule along the $z$-axis of the laboratory frame. The degree of orientation is given here by $\left\langle J_{z}\right\rangle / \sqrt{\left\langle J^{2}\right\rangle}$, where $J_{z}$ is the $z$-component of the angular momentum $J$. This aspect has been recently studied in a series of works, both theoretically [43, 44, 45] and experimentally [46, 47, 48. In particular, it has been shown in Ref. [45] that orientation of the angular momentum can be achieved by a sequence of two short laser pulses, properly delayed and polarized at 45 degrees with respect to each other. Here we revisit this control problem using the two monotonically convergent algorithms. An elliptical polarization is considered to realize this orientation. The corresponding Hamiltonian is given by Eq. (5). Since the angular momentum of a diatomic molecule is classically orthogonal to the molecular axis, its alignment along the laboratory $z$ - axis is equivalent to a delocalization of the molecular axis in the $(x, y)$ - plane. This delocalization can also be interpreted as a minimization of the expectation value $\left\langle\cos ^{2} \theta\right\rangle$ [4].

Let us consider a state of the form $|j, \pm j\rangle$. Straightforward computation 


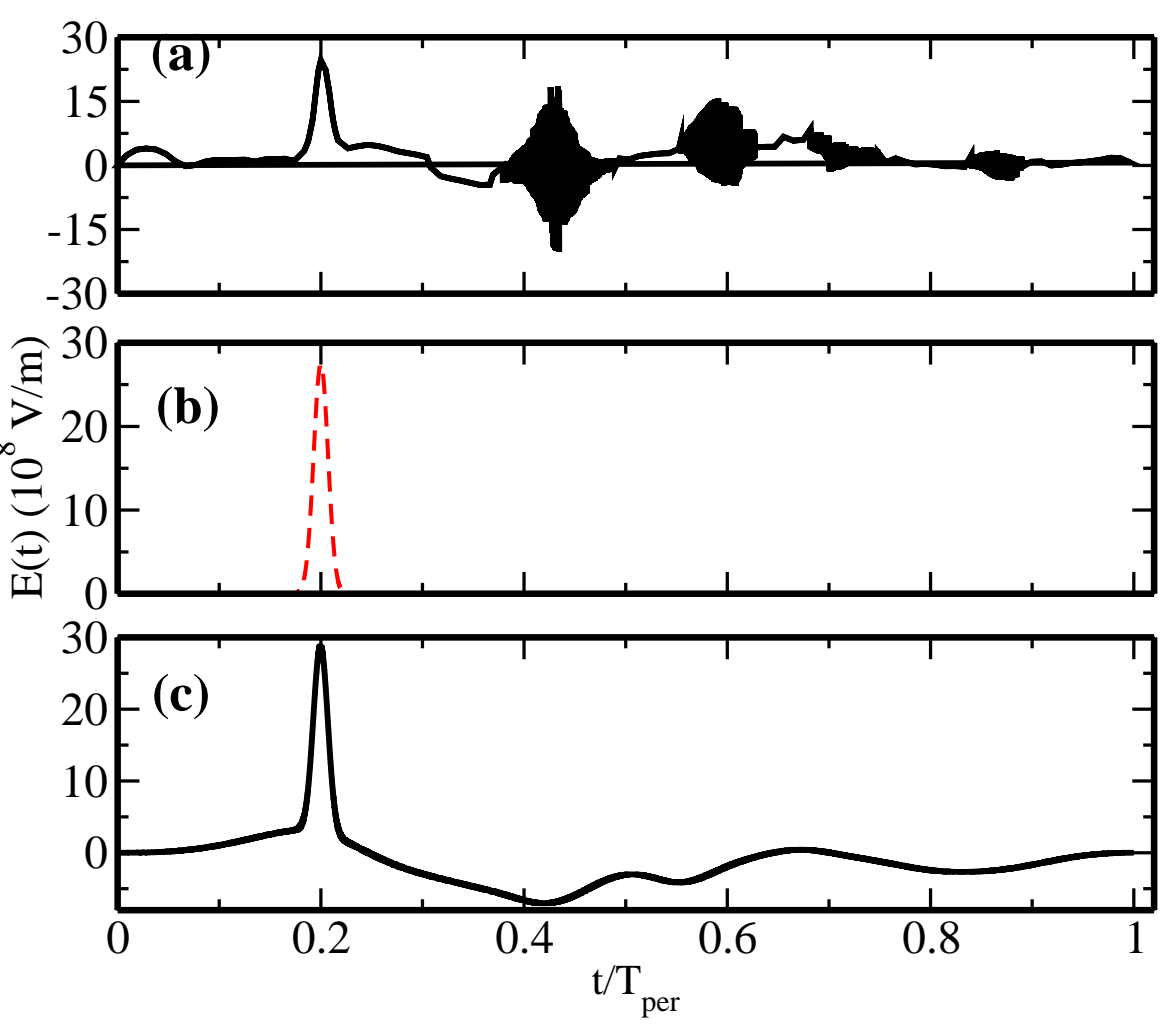

Figure 3: (color online) Comparison of the optimized pulses obtained from the Krotov (c) and Lapert (a) algorithms. The panel (b) depicts the initial field used for the two algorithms.

shows that

$$
\left\langle j, \pm j\left|\cos ^{2} \theta\right| j, \pm j\right\rangle=\frac{1}{2 j+3} .
$$

When $j \rightarrow \infty$, the right hand side of Eq. (10) converges to its minimum value, 0 . Therefore, in a sub-Hilbert space of finite dimension, the states $|j, \pm j\rangle$ minimize $\left\langle\cos ^{2} \theta\right\rangle$ for large $j$. In other words, these states maximize the delocalization of the molecular axis in the $(x, y)$-plane. Consequently, the states $|j, j\rangle$ and the states $|j,-j\rangle$ maximize and minimize $\left\langle J_{z}\right\rangle$, respectively [45].

At $T=0 \mathrm{~K}$, the initial state is $|0,0\rangle$ and $|4,4\rangle$ is taken as the target state. The minimum expectation value that can be reached with this choice is of the order of $\left\langle 4, \pm 4\left|\cos ^{2} \theta\right| 4, \pm 4\right\rangle \approx 0.1$. Note that the more we increase $j$, the better the delocalization becomes. However, both difficulty and numerical cost increase with $j$. Therefore, target states of the form $|j, \pm j\rangle$ with $j>4$ will not be analyzed.

When the relative phase $\Phi_{x}-\Phi_{y}$ is chosen so that the cross term of the Hamiltonian (5) vanishes, the dynamics cannot distinguish the states $|j, j\rangle$ and $|j,-j\rangle$ (see [44] for the analytical proof). Here, in order to get a completely controllable system, the relative phase $\Phi_{x}-\Phi_{y}$ is set to $\pi / 4$. The guess field is constructed as a series of Gaussian pulses of $150 \mathrm{fs}$ FWHM for each component, $\epsilon_{x}$ and $\epsilon_{y}$. We have chosen $\lambda^{\mathrm{Lp}}=10^{5}$ and $\lambda^{\mathrm{Kr}}=0.1$. Our choice of a large $\lambda$ 

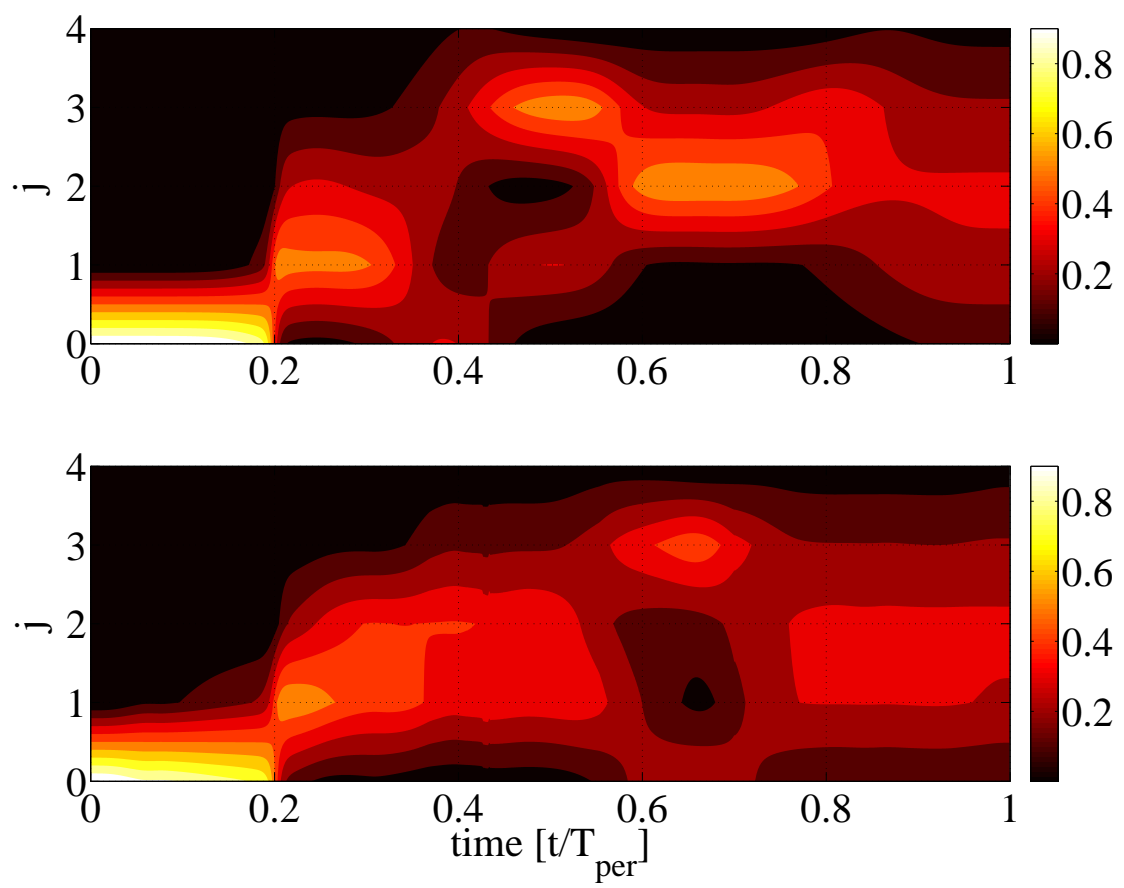

Figure 4: (color online) Projection of the time-dependent wave function onto the eigenstates of the system. The top and bottom panels represent respectively the dynamics induced by the pulses optimized with the Krotov and Lapert algorithms.

value for the Lapert algorithm is motivated by the fact that small values induce very fast oscillations of the optimized control field, which are physically and numerically not very interesting. For 50 iterations, the target state is reached with a probability of the order of 0.99 for the two algorithms. The convergence of the cost $C_{t_{f}}$ (top panel), plotted as a function of the number of iterations, is shown in Fig. 5 for the two algorithms. The corresponding optimized pulses are shown in the bottom panels. The convergence to the target state is similar to the one observed in the first example. In particular, the Lapert algorithm converges faster initially, the Krotov algorithm remaining more efficient, specifically in terms of CPU time. In addition, Fig. 5 clearly shows that the structure of the Krotov solution is simpler than the Lapert one.

\subsection{Control of a thermal rotational sample}

The last example concerns the control of a thermal sample at non-zero temperature. The initial state is given by the Boltzmann distribution, which can be written as follows:

$$
\rho_{0}=\frac{1}{Z} \sum_{j} \sum_{m=-j}^{j} e^{-\frac{B j(j+1)}{k_{B} T}}|j, m\rangle\langle j, m|,
$$



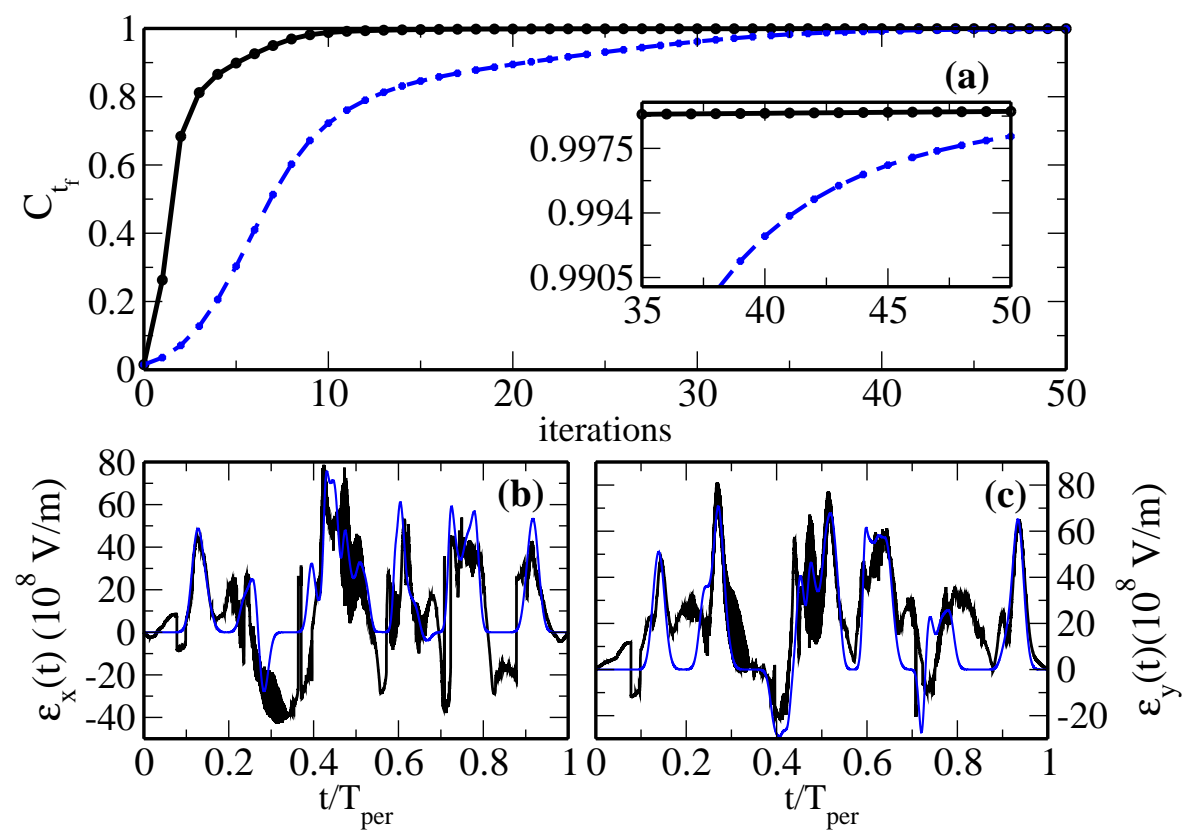

Figure 5: (Color online) (a): Convergence of the Lapert and the Krotov algorithms measured by the final cost $C_{t_{f}}$. $C_{t_{f}}=\left|\left\langle 4,4 \mid \psi\left(t_{f}\right)\right\rangle\right|^{2}$ (top panel) is plotted as a function of the number of iterations. (b): $x$-components of the optimized fields. (c): $y$ - components the optimized fields. The black and blue (dark gray) curves depict respectively the results derived with the Lapert and the Krotov algorithms.

where $T$ is the temperature, $k_{B}$ the Boltzmann constant and $Z$ the partition function, which is expressed as

$$
Z=\sum_{j} \sum_{m=-j}^{j} e^{-\frac{B j(j+1)}{k_{B} T}} .
$$

The control aims at reaching the state that maximizes the permanent alignment of the molecule along the $z$-axis. The molecular alignment is measured by the expectation value $\left\langle\cos ^{2} \theta\right\rangle$, which can be written as the sum of two terms:

$$
\left\langle\cos ^{2} \theta\right\rangle=\left\langle\cos ^{2} \theta\right\rangle_{p}+\left\langle\cos ^{2} \theta\right\rangle_{c},
$$

where $\left\langle\cos ^{2} \theta\right\rangle_{p}=\sum_{j, m, m^{\prime}} \rho_{j m, j m^{\prime}} C_{j m, j m^{\prime}}$ and $\left\langle\cos ^{2} \theta\right\rangle_{c}=\sum_{j \neq j^{\prime}, m, m^{\prime}} \rho_{j m, j^{\prime} m^{\prime}} C_{j m, j^{\prime} m^{\prime}}$. The coefficients $C_{j m, j^{\prime} m^{\prime}}$ denote the matrix elements of the operator $\cos ^{2} \theta$. Partitioning the alignment measured into diagonal and off-diagonal terms (with respect to the quantum number $j$ ) reveals interesting physical information about the rotational dynamics. While $\left\langle\cos ^{2} \theta\right\rangle_{p}$ provides a direct measure of the rotational population, $\left\langle\cos ^{2} \theta\right\rangle_{c}$ leads to the temporal evolution of the coherences. By definition, $\left\langle\cos ^{2} \theta\right\rangle_{p}$ is constant when the pulse is switched off. In some applications, it can be interesting to maximize only the permanent alignment.

For this purpose, we use the strategy proposed in Ref. [40. Considering a sub-Hilbert space $\mathcal{H}_{j_{f}}$ of finite dimension defined by the condition $j \leq j_{f}$, we 
introduce the diagonal projection of the $\cos ^{2} \theta$ operator:

$$
\cos ^{2} \theta_{p}=\sum_{j, m, m^{\prime}}|j, m\rangle\left\langle j, m\left|\cos ^{2} \theta\right| j, m^{\prime}\right\rangle\left\langle j, m^{\prime}\right|,
$$

such that $\left\langle\cos ^{2} \theta_{p}\right\rangle=\left\langle\cos ^{2} \theta\right\rangle_{p}$. The target state $\rho_{f}$ of the control problem is defined as the density matrix maximizing $\left\langle\cos ^{2} \theta_{p}\right\rangle$ and reachable from the initial state $\rho_{0}$. Due to the constraint of unitary evolution, the density matrices $\rho_{0}$ and $\rho_{f}$ have the same spectrum. In addition, it can be shown that, in this subspace, the two operators $\cos ^{2} \theta_{p}$ and $\rho_{f}$ can be simultaneously diagonalized. One therefore deduces that

$$
\max \left[\left\langle\cos ^{2} \theta_{p}\right\rangle\right]=\sum_{k=1}^{N} \chi_{k} \omega_{k}
$$

where $\chi_{1} \leq \chi_{2} \leq \cdots \leq \chi_{N}$ and $\omega_{1} \leq \omega_{2} \leq \cdots \leq \omega_{N}$ are the eigenvalues of $\cos ^{2} \theta_{p}$ and $\rho_{f}$, respectively. The integer $N$ is the dimension of $\mathcal{H}_{j_{f}}$. If we denote by $\left|\chi_{k}\right\rangle$ the eigenvectors of $\cos ^{2} \theta_{p}, \rho_{f}$ becomes

$$
\rho_{f}=\sum_{k=1}^{N} \omega_{k}\left|\chi_{k}\right\rangle\left\langle\chi_{k}\right| .
$$

Since the subspaces of a given parity of $j$ are not coupled by the operators $\cos ^{2} \theta_{x, y, z}$, the subdivision $\mathcal{H}_{j_{f}}=\mathcal{H}_{j_{f}}^{(\text {even })} \oplus \mathcal{H}_{j_{f}}^{(\text {odd })}$ has to be considered to properly define the target state, see Ref. [40] for details of this construction.

Figure [ displays the partial trace $\operatorname{Tr}_{j}[\cdot]$ with respect to $j$ of the target state $\rho_{f}$, with $j_{f}=4$, for the CO molecule at $T=5 \mathrm{~K}$. For a given value $m$, this trace is defined by $\sum_{j=|m|}^{j_{\max }} \rho_{j m, j m}^{2}$. For comparison, we have also plotted the same distribution for the initial state $\rho_{0}$. One clearly sees in Fig. [6 that the optimal distribution is narrowed compared to the thermal one. Since $\rho_{f}$ is a diagonal matrix, there is no coherence and we get $\left\langle\cos ^{2}\right\rangle_{c}=0$. At $T=0$ $\mathrm{K}$, the maximum permanent alignment is equal to 0.6. This maximum is a temperature-dependent function, and for $T \neq 0 \mathrm{~K}, \max \left[\left\langle\cos ^{2} \theta_{p}\right\rangle\right] \leq 0.6$. For example at $T=5 \mathrm{~K}, \max \left[\left\langle\cos ^{2} \theta_{p}\right\rangle\right]=0.518$.

We use Eq. (5) and the same guess pulse as in Sec. 3.2. The parameter $j_{f}$ is fixed to 4 . We have chosen $\lambda=2 \times 10^{-2}$ and $5 \times 10^{5}$ for the Krotov and Lapert algorithms, respectively. Figure 7 compares the permanent alignment dynamics for the two algorithms at $T=5 \mathrm{~K}$. The pulse is switched off at $t_{f}=T_{p e r}$. The dynamics are found to be step-like, such that $\left\langle\cos ^{2} \theta\right\rangle_{p}$ is either constant or varies suddenly. A permanent alignment of the order of 0.47 and 0.49 is reached for the Lapert and the Krotov algorithms, respectively for 500 iterations. In this example, the comparison of the convergence measured by the final cost is not shown. We observe as in Fig. 2 the same behavior for the Lapert and Krotov algorithms. As illustrated in Fig. 7, the optimized solution designed by the Krotov algorithm is simpler than the one given by the Lapert algorithm.

\section{Summary}

For key control problems of the rotational dynamics, we have designed in this work different optimized pulses from two monotonically convergent algorithms, 


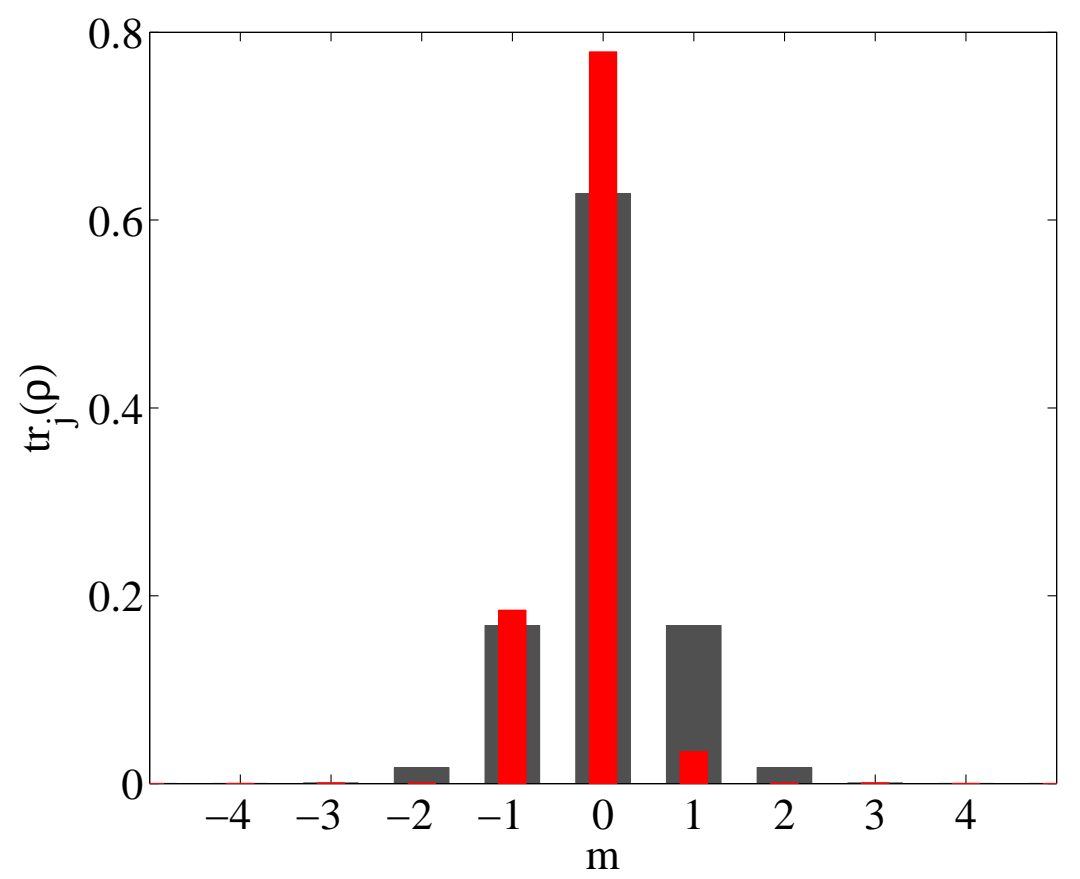

Figure 6: (color online) Partial trace $\operatorname{Tr}_{j}[\cdot]$ with respect to the quantum number $j$ of initial and target state distributions at $T=5 \mathrm{~K}$. The target distribution corresponds to $\rho_{f}$, (red or dark gray bar) which is the density matrix maximizing the permanent alignment at $T=5 \mathrm{~K}$. The partial trace of the initial density matrix is in gray. The parameter $j_{f}$ is set to 4 .

the Lapert and the Krotov ones. Our numerical findings confirmed by the three examples discussed in this work are as follows:

1. The final fidelities reached by the two algorithms are very similar.

2. The Lapert algorithm is somehow more flexible compared to the Krotov one in the sense that the parameter $\lambda$ can be chosen without any constraints, while this parameter has to satisfy the relation (29) in the Krotov approach in order to ensure monotonic convergence.

3. The Krotov algorithm is more efficient than the Lapert one in terms of CPU time.

4. The optimized Krotov field has a simpler structure than the Lapert one, which generally presents some unwanted oscillatory behaviors.

Having in mind the work of Ref. [49], an open question is now the application of these optimal control algorithms to more complex systems. The computation of the optimal field allowing the cooling of rovibrational dynamics could be an interesting test case, in particular because non unitary processes have to be taken into account. 

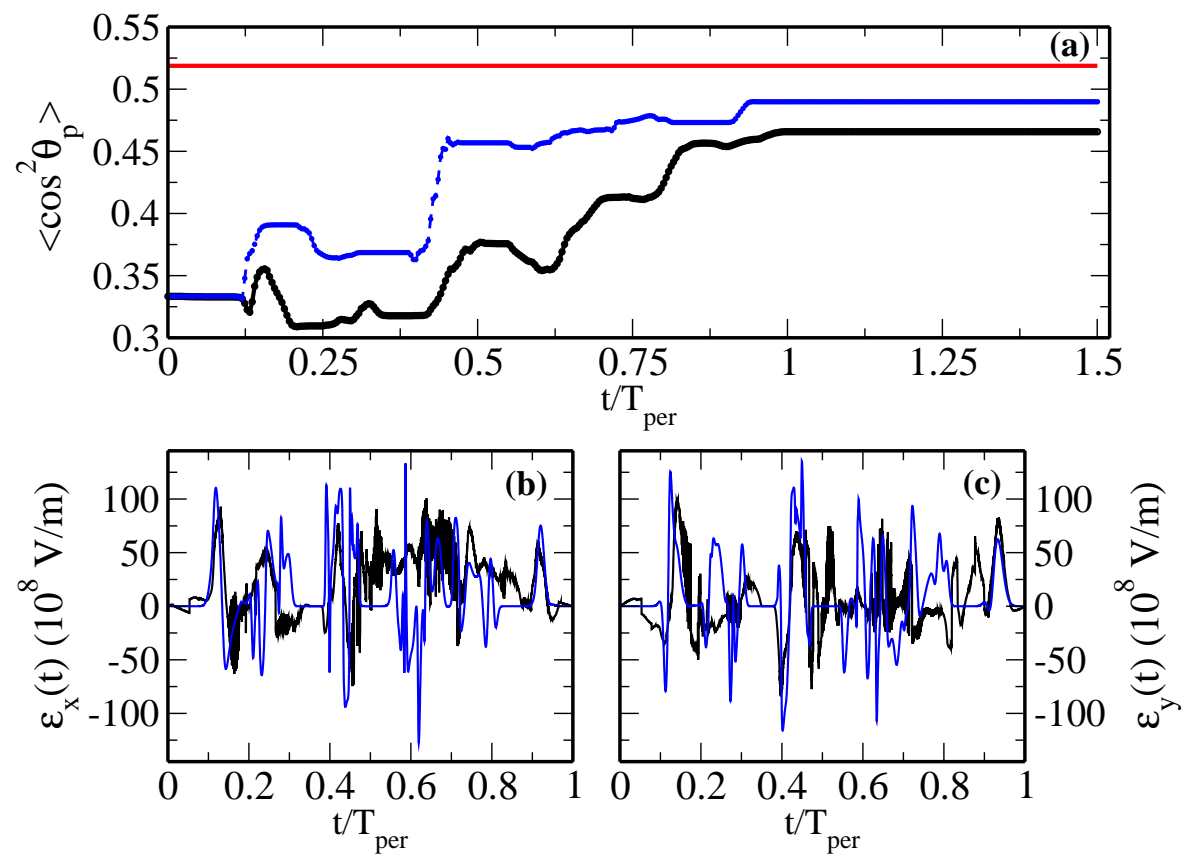

Figure 7: (Color online) (a): Permanent alignment dynamics during and after the application of the optimized pulses. The black and blue (dark gray) solid lines correspond to the dynamics generated by the Lapert and the Krotov optimized pulses. The red (light gray) horizontal solid line depicts here the maximum permanent alignment that can be reached in the subspace $\mathcal{H}_{j_{f}}$. (b): $x$-Components of the optimized fields. (c): $y$-Components the optimized fields. The black and blue (dark gray) curves depict respectively the results derived with the Lapert and the Krotov algorithms.

\section{Acknowledgment}

The authors thank Daniel Reich for many helpful discussions and his comments on the manuscript. Financial supports from the Conseil Régional de Bourgogne and the QUAINT coordination action (EC FET-Open) are gratefully acknowledged.

\section{A Description of monotonically convergent algo- rithms}

The Krotov and Lapert optimization algorithms are summarized here for pure state quantum dynamics. This description is straightforwardly extended to the density matrix formalism. To simplify notation, we restrict ourself to the maximization of the projection onto a target state. The two algorithms can analogously be used for maximization or minimization of the expectation value of a given observable. The control problem is characterized by maximization of 
the functional $C$,

$$
C=C_{t_{f}}\left[\left\{\left|\psi\left(t_{f}\right)\right\rangle\right\}\right]-\int_{0}^{t_{f}} C_{t}[\{|\psi(t)\rangle\}, E(t)] d t,
$$

where $C_{t_{f}}$ is the final time cost functional and $C_{t}$ the running cost. The parameter $E$ denotes the external field and $|\psi(t)\rangle$ the wave function describing the state of the system at time $t$. Its time evolution is governed by Eq. (6). If $\left|\psi_{f}\right\rangle$ is the target state, the final cost can be defined as follows:

$$
C_{t_{f}}\left[\left\{\left|\psi\left(t_{f}\right)\right\rangle\right\}\right]=\left|\left\langle\psi_{f} \mid \psi\left(t_{f}\right)\right\rangle\right|^{2} .
$$

Here, only a running cost which does not depend on the state of the system is considered:

$$
C_{t}[\{|\psi(t)\rangle\}, E(t)]=g[E(t)] .
$$

Extension to a state-dependent running cost is described in Ref [19]. The main difference between the two algorithms is in the choice of the running cost.

\section{A.1 Krotov's method}

The derivation of the Krotov algorithm presented here follows closely Ref [19], specializing it to a non-linear interaction of the system with the control field, see Eqs. (21) and (5). There is no requirement for a specific power of $E(t)$ the running cost $g$ so we choose it to minimize the change in the energy of the field [21],

$$
g[E(t)]=\frac{\lambda}{S(t)}\left(E(t)-E_{r e f}\right)^{2},
$$

with $E_{r e f}$ denoting a reference field, $S(t)$ a shape function and $\lambda$ a weight.

Krotov's method is based on the construction of an auxiliary functional, $\mathcal{L}[\{|\psi\rangle\}, E, \Phi]$ with $\Phi$ an arbitrary functional. It is chosen such that the maximization of $\mathcal{L}$ is equivalent to maximization of $C$ of Eq (17). $\Phi$ is used to ensure a global minimum with respect to changes in the state. Then any change in the state will lead to an increase in the value of $\mathcal{L}$, i.e., to monotonic convergence [16, 20]. This is achieved by expanding $\Phi$ to second order in the change of the state, $|\Delta \psi(t)\rangle$,

$$
\begin{aligned}
\Phi[\{|\psi\rangle\}, t]= & \langle\chi(t) \mid \psi(t)\rangle+\langle\psi(t) \mid \chi(t)\rangle \\
& +\frac{1}{2}\langle\Delta \psi(t)|\sigma(t)| \Delta \psi(t)\rangle .
\end{aligned}
$$

When the equations of motion are linear with respect to the state and the running cost functional does not depend on the state, the second order contribution is not required [19]. Since, in this work, we consider a quantum control problem which fulfills these conditions, a first order construction of $\Phi$ is sufficient. The construction of $\Phi$ is described in detail in Ref. [19]. The auxiliary functional is defined by:

$$
\begin{aligned}
\mathcal{L}[\{|\psi\rangle\}, E, \Phi]= & G\left[\left\{\left|\psi\left(t_{f}\right)\right\rangle\right\}\right]-\Phi[\{|\psi(0)\rangle\}, 0] \\
& -\int_{0}^{t_{f}} R[\{|\psi(t)\rangle\}, E(t), t] d t
\end{aligned}
$$


where the final time functional and running functional, $G$ and $R$, are given by

$$
\begin{gathered}
G\left(\left\{\left|\psi\left(t_{f}\right)\right\rangle\right\}\right)=C_{t_{f}}\left[\left\{\left|\psi\left(t_{f}\right)\right\rangle\right\}\right]+\Phi\left[\left\{\left|\psi\left(t_{f}\right)\right\rangle\right\}, t_{f}\right], \\
R[\{|\psi(t)\rangle\}, E(t), t]=\frac{\partial \Phi}{\partial t}+g[E(t)]-i\left(\nabla_{|\psi\rangle} \Phi\right)|H \psi(t)\rangle \\
\\
+i\langle\psi(t)| H\left(\nabla_{\langle\psi|} \Phi\right)
\end{gathered}
$$

For a maximization problem, the following conditions have to be fulfilled:

$$
\mathcal{L}\left[\left\{\left|\psi_{k}\right\rangle\right\}, E_{k}, \Phi\right] \leq \mathcal{L}\left[\left\{\left|\psi_{k+1}\right\rangle\right\}, E_{k+1}, \Phi\right],
$$

where $k$ indicates the iterative step. Sufficient conditions for maximizing $\mathcal{L}$ translate into maximizing $G$ and minimizing $R$ at each time:

$$
\begin{gathered}
\mathcal{L}\left[\left\{\left|\psi_{k+1}\right\rangle\right\}, E_{k}, \Phi\right]-\mathcal{L}\left[\left\{\left|\psi_{k}\right\rangle\right\}, E_{k+1}, \Phi\right]= \\
\Delta_{1}+\int_{0}^{t_{f}} \Delta_{2}(t) d t+\int_{0}^{t_{f}} \Delta_{3}(t) d t
\end{gathered}
$$

where the $\Delta_{i}, i=1, \cdots, 3$ are given by:

$$
\begin{aligned}
& \Delta_{1}=G\left[\left\{\left|\psi_{k+1}\left(t_{f}\right)\right\rangle\right\}\right]-G\left[\left\{\left|\psi_{k}\left(t_{f}\right)\right\rangle\right\}\right], \\
& \Delta_{2}(t)= R\left[\left\{\left|\psi_{k+1}(t)\right\rangle\right\}, E_{k}(t), t\right] \\
&-R\left[\left\{\left|\psi_{k+1}(t)\right\rangle\right\}, E_{k+1}(t), t\right],
\end{aligned}
$$

and

$$
\begin{aligned}
\Delta_{3}(t)= & R\left[\left\{\left|\psi_{k}(t)\right\rangle\right\}, E_{k}(t), t\right] \\
& -R\left[\left\{\left|\psi_{k}(t)\right\rangle\right\}, E_{k+1}(t), t\right] .
\end{aligned}
$$

Non-negativeness of the $\Delta_{i}(i=1, \cdots, 3)$ ensures monotonic convergence. For a quantum control problem where the cost functional is state-independent and the equations of motion are linear with respect to the state, positivity of $\Delta_{1}$ and $\Delta_{3}$ is automatically satisfied [20, 21]. Non-negativeness of $\Delta_{2}$ can be obtained by a proper choice of $\lambda$ and the shape function $S(t)$ [19]:

$$
\frac{\lambda}{S(t)}>\Delta_{M_{2}^{E}}
$$

where $\Delta_{M_{2}^{E}}$ is the spectral radius of $M_{2}^{E}=\partial^{2} H[E(t)] / \partial E^{2}$.

Evaluating the extremum condition for $\mathcal{L}$ yields the control equations,

1. Equation of the control field:

$$
\begin{aligned}
\left.\frac{\partial g}{\partial E}\right|_{E_{k+1}}(t) & = \\
& 2 \mathfrak{I m}\left[\left\langle\chi_{k}(t)\left|\frac{\partial H[E(t)]}{\partial E}\right|_{E_{k+1}} \mid \psi_{k+1}(t)\right\rangle\right] .
\end{aligned}
$$


2. Equation of motion for the adjoint state $|\chi\rangle$, with 'initial' condition:

$$
\begin{aligned}
i \frac{\partial}{\partial t}\left|\chi_{k}(t)\right\rangle & =H\left[E_{k}(t)\right]\left|\chi_{k}(t)\right\rangle \\
\left|\chi_{k}(t=T)\right\rangle & =-\left.\nabla_{\left\langle\psi_{k}\right|} C_{t_{f}}\right|_{\left|\psi_{k}\right\rangle} .
\end{aligned}
$$

3. Equation of motion of the state $|\psi\rangle$, with initial condition $\left|\psi_{i n i}\right\rangle$

$$
\begin{aligned}
i \frac{\partial}{\partial t}\left|\psi_{k+1}(t)\right\rangle & =H\left[E_{k+1}(t)\right]\left|\psi_{k+1}(t)\right\rangle \\
\left|\psi_{k+1}(t=0)\right\rangle & =\left|\psi_{i n i}\right\rangle .
\end{aligned}
$$

At each iteration, the update of the field $E_{k+1}(t)$ obtained from Eq. (30) involves a backward and a forward propagation, Eq. (31) and Eq. (33), respectively. If the system interacts non-linearly with the control field, both left and right hand sides of Eq. (30) depend on $E_{k+1}(t)$. For simplicity, we assume that the change of the control field between iterations $k$ and $k+1$ is small enough such that $\partial H(E(t)) /\left.\partial E\right|_{E_{k+1}} \approx \partial H(E(t)) /\left.\partial E\right|_{E_{k}}$.

\section{A.2 The Lapert approach}

\section{A.2.1 General description}

While in the Krotov method, this running cost minimizes the change in the energy of the field, in the Lapert algorithm, this choice is different. Basically, $g$ is chosen so as Eq. (30) admits a real solution at any time $t$. The cost is defined as follows:

$$
g[E(t)]=\frac{\lambda}{S(t)}\left(E(t)-E_{r e f}\right)^{2 n},
$$

For a Hamiltonian given by Eq. (2), Eq. (30) leads to

$$
\begin{aligned}
& 2 n \frac{\lambda}{S(t)}\left(E_{k+1}(t)-E_{k}(t)\right)^{2 n-1}- \\
& 2 \Im\left[\left\langle\chi_{k}\left|\mu+2 \alpha E_{k+1}+3 \beta E_{k+1}^{2}\right| \psi_{k+1}\right\rangle\right]=0
\end{aligned}
$$

The left hand side of Eq. (36) can be viewed as a polynomial of $E_{k+1}$. Choosing the integer $n$ such that $\lambda / S(t) E_{k+1}^{2 n-1}$ is a monomial of order higher than the right hand side of Eq. (36) ensures that there exists a real solution to the equation at each time $t$. For a non-linearity of order $3, n=2$ is sufficient. The conditions for monotonic convergence are determined through variation of $\Delta C$ given by:

$$
\begin{aligned}
\Delta C= & C_{k+1}-C_{k} \\
= & C_{t_{f}}\left[\left\{\left|\psi_{k+1}\left(t_{f}\right)\right\rangle\right\}\right]-C_{t_{f}}\left[\left\{\left|\psi_{k}\left(t_{f}\right)\right\rangle\right\}\right] \\
& +\int_{0}^{t_{f}} g\left[E_{k+1}(t)\right]-g\left[E_{k}(t)\right] d t .
\end{aligned}
$$

which needs to be positive [25]. 


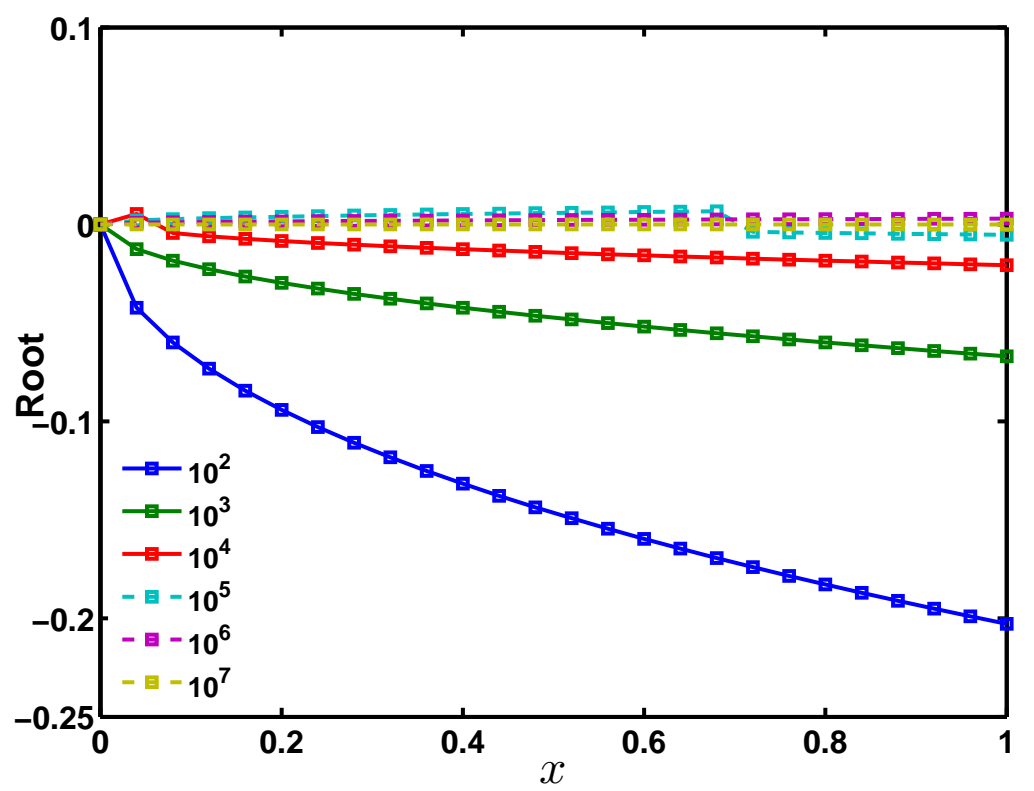

Figure 8: (Color online) Variation of one of the real roots of Eq. (38) as a function of $x=\langle\chi(t) \mid \psi(t)\rangle$ for different values of $\lambda, 10^{2} \leq \lambda \leq 10^{7}$.

\section{A.2.2 Role of the parameter $\lambda$}

We discuss in this section the way the parameter $\lambda$ affects the optimized solution. For this purpose, we have analyzed the behavior of one of the real roots of the polynomial Eq. (36). To simplify the description, the operators $\vec{\mu}, \alpha$ and $\beta$ have been replaced by their maximum eigenvalues. For $n=2$, Eq. (36) can then be written as follows:

$$
\begin{aligned}
& 4 \frac{\lambda}{S(t)}\left(E_{k+1}(t)-E_{k}(t)\right)^{3}-6|\beta|_{\max } \Im\left[\left\langle\chi_{k} \mid \psi_{k+1}\right\rangle\right] E_{k+1}^{2} \\
& -4|\alpha|_{\max } \Im\left[\left\langle\chi_{k} \mid \psi_{k+1}\right\rangle\right] E_{k+1} \\
& -2|\mu|_{\max } \Im\left[\left\langle\chi_{k} \mid \psi_{k+1}\right\rangle\right]=0 .
\end{aligned}
$$

Figure 8illustrates the variation of one of the real roots of Eq. (38) as a function of $x=\langle\chi(t) \mid \psi(t)\rangle$ for different values of $\lambda$. The range of $\lambda$ is taken from $10^{2}$ to $10^{7}$. For large values of $\lambda$, the variation of the root is very slow with respect to $x$ while for a value smaller then $10^{4}$, the change of the roots can be very fast. This observation qualitatively explains the fast oscillations occurring in the Lapert optimized fields.

\section{References}

[1] L. Pontryagin et al., Mathematical theory of optimal processes, Mir, Moscou, 1974. 
[2] S. Rice and M. Zhao, Optimal control of molecular dynamics (Wiley, New York, 2003).

[3] M. Shapiro and P. Brumer, Principles of quantum control of molecular processes (Wiley, New York, 2003).

[4] D. J. Tannor : Introduction to quantum mechanics: A time-dependent perspective, (University Science Books, Sausalito 2007).

[5] R. C. C. Brif and H. Rabitz, New J. Phys. 12, 075008 (2010).

[6] N. Khaneja, R. Brockett and S. J. Glaser, Phys. Rev. A, 63, 032308 (2001).

[7] M. Lapert, Y. Zhang, M. Braun, S. J. Glaser and D. Sugny, Phys. Rev. Lett. 104, 083001 (2010); M. Lapert, Y. Zhang, M. Braun, S. J. Glaser and D. Sugny, Phys. Rev. A 82, 063418 (2010).

[8] E. Assémat, M. Lapert, Y. Zhang, M. Braun, S. J. Glaser and D. Sugny, Phys. Rev. A 82, 013415 (2010).

[9] R. Kosloff, S. A. Rice, P. Gaspard, S. Tersigni and D. Tannor, Chem. Phys. 139, 201 (1989).

[10] J. Somlói, V. A. Kazakovski, and D. J. Tannor, Chem. Phys. 172, 85 (1993).

[11] W. Zhu, J. Botina, and H. Rabitz, J. Chem. Phys. 108, 1953 (1998).

[12] T. E. Skinner, T. O. Reiss, B. Luy, N. Khaneja and S. J. Glaser, J. Magn. Reson. 163, 8 (2003); T. E. Skinner, T. O. Reiss, B. Luy, N. Khaneja and S. J. Glaser, J. Magn. Reson. 172, 17 (2005).

[13] Y. Maday and G. Turinici, J. Chem. Phys. 118, 8191 (2003).

[14] Y. Ohtsuki, G. Turinici and H. Rabitz, J. Chem. Phys. 120, 5509 (2004).

[15] J. Werschnik and E. K. U. Gross, J. Phys. B 40, R175 (2007).

[16] A. I. Konnov and V. F. Krotov, Automation and Remote Control 60, 1427 (1999).

[17] V. F. Krotov, Doklady Mathematics 78, 949 (2008).

[18] V. F. Krotov, Automation and Remote Control, 70, 357 (2009).

[19] D. M. Reich, M. Ndong and C. P. Koch, J. Chem. Phys. 136, 104103 (2012).

[20] S. E. Sklarz and D. J. Tannor, Phys. Rev. A 66, 053619 (2002).

[21] J. P. Palao and R. Kosloff, Phys. Rev. A 68, 062308 (2003).

[22] C. P. Koch, J. P. Palao, R. Kosloff and F. Masnou-Seeuws, Phys. Rev. A 70, 013402 (2004).

[23] J. P. Palao, R. Kosloff and C. P. Koch, Phys. Rev. A 77, 063412 (2008).

[24] Y. Ohtsuki and K. Nakagami, Phys. Rev. A 77, 033414 (2008). 
[25] M. Lapert, R. Tehini, G. Turinici and D. Sugny, Phys. Rev. A 78, 023408 (2008).

[26] B. Friedrich and D. Herschbach, Phys. Rev. Lett. 74, 4623 (1995).

[27] T. Seideman and E. Hamilton, Adv. At. Mol. Opt. Phys. 52, 289 (2006).

[28] H. Stapelfeldt and T. Seideman, Rev. Mod. Phys. 75, 543 (2003).

[29] M. Leibscher, I. S. Averbukh and H. Rabitz, Phys. Rev. Lett. 90, 213001 (2003).

[30] J. Salomon, C. M. Dion and G. Turinici, J. Chem. Phys. 123, 144310 (2005).

[31] S.-L. Liao, T.-S. Ho, H. Rabitz and S.-I. Chu, Phys. Rev. A 87, 013429 (2013).

[32] T. Viellard, F. Chaussard, D. Sugny, B. Lavorel and O. Faucher, J. Raman Spec. 39, 694 (2008).

[33] M. Lapert and D. Sugny, Phys. Rev. A 85, 063418 (2012).

[34] T. Kanai and H. Sakai, J. Chem. Phys. 115, 5492 (2001).

[35] R. Tehini and D. Sugny, Phys. Rev. A, 77, 023407 (2008).

[36] H. Abe, Y. Ohtsuki, Phys. Rev. A 83, 053410 (2011); K. Nakagami, Y. Mizumoto and Y. Ohtsuki, J. Chem. Phys. 129, 194103 (2008); H. Abe, Y. Ohtsuki, Chem. Phys. 400, 13 (2012); K. Nakajima.

[37] H. Abe and Y. Ohtsuki, J. Phys. Chem. A 116, 11219 (2012).

[38] M. D. Feit, J. A. Fleck and A. Steiger, J. Comput. Phys. 47, 412 (1982).

[39] D. Sugny, A. Keller, O. Atabek, D. Daems, C. M. Dion, S. Guérin and H. R. Jauslin, Phys. Rev. A 71, 063402 (2005); D. Sugny and M. Joyeux, J. Chem. Phys. 112, 31 (2000).

[40] D. Sugny, A. Keller, O. Atabek, D. Daems, C. M. Dion, S. Guérin and H. R. Jauslin, Phys. Rev. A 72, 032704 (2005).

[41] C. Gollub, M. Kowalewski and R. de Vivie-Riedle, Phys. Rev. Lett. 101, 073002 (2008).

[42] M. Lapert, R. Tehini, G. Turinici and D. Sugny, Phys. Rev. A 79, 063411 (2009).

[43] S. Fleischer et al., New J. of Phys. 11, 105039 (2009).

[44] M. Lapert, E. Hertz, S. Guérin and D. Sugny, Phys. Rev. A 80, 051403 (2009).

[45] M. Lapert, S. Guérin and D. Sugny, Phys. Rev. A 83, 013403 (2011).

[46] K. Kitano, H. Hasegawa and Y. Ohshima, Phys. Rev. Lett. 103, 223002 (2009). 
[47] M. Z. Hoque, M. Lapert, E. Hertz, F. Billard, D. Sugny, B. Lavorel and O. Faucher, Phys. Rev. A 84, 013409 (2011).

[48] S. Zhdanovich, A. A. Milner, C. Bloomquist, J. Flob, I. Sh. Averbukh, J. W. Hepburn and V. Milner, Phys. Rev. Lett. 107, 243004 (2011).

[49] I. Manai, R. Horchani, H. Lignier, P. Pillet, D. Comparat, A. Fioretti and M. Allegrini, Phys. Rev. Lett. 109, 183001 (2012) 